\title{
Vascular access for renal replacement therapy among 459 critically ill patients: a pragmatic analysis of the randomized AKIKI trial
}

Nicolas Benichou ${ }^{1,28,29}$ D , Saïd Lebbah² ${ }^{2}$ David Hajage ${ }^{2,3,4}$, Laurent Martin-Lefèvre ${ }^{5}$, Bertrand Pons ${ }^{6}$, Eric Boulet ${ }^{7}$, Alexandre Boyer ${ }^{8}$, Guillaume Chevrel ${ }^{9}$, Nicolas Lerolle ${ }^{10}$, Dorothée Carpentier ${ }^{11}$, Nicolas de Prost ${ }^{12,13}$, Alexandre Lautrette ${ }^{14}$, Anne Bretagnol $^{15}$, Julien Mayaux ${ }^{16}$, Saad Nseir ${ }^{17}$, Bruno Megarbane ${ }^{18}$, Marina Thirion ${ }^{19}$, Jean-Marie Forel ${ }^{20}$, Julien Maizel ${ }^{21}$, Hodane Yonis ${ }^{22}$, Philippe Markowicz ${ }^{23}$, Guillaume Thiery ${ }^{6}$, Frederique Schortgen ${ }^{24}$, Florence Tubach ${ }^{2,3,25}$, Jean-Damien Ricard ${ }^{26,27}$, Didier Dreyfuss ${ }^{27,28,29 \dagger}$ and Stéphane Gaudry $29,30,31 *+$

\begin{abstract}
Background: Vascular access for renal replacement therapy (RRT) is routine question in the intensive care unit. Randomized trials comparing jugular and femoral sites have shown similar rate of nosocomial events and catheter dysfunction. However, recent prospective observational data on RRT catheters use are scarce. We aimed to assess the site of RRT catheter, the reasons for catheter replacement, and the complications according to site in a large population of critically ill patients with acute kidney injury.

Patients and methods: We performed an ancillary study of the AKIKI study, a pragmatic randomized controlled trial, in which patients with severe acute kidney injury (KDIGO 3 classification) with invasive mechanical ventilation, catecholamine infusion or both were randomly assigned to either an early or a delayed RRT initiation strategy. The present study involved all patients who underwent at least one RRT session. Number of RRT catheters, insertion sites, factors potentially associated with the choice of insertion site, duration of catheter use, reason for catheter replacement, and complications were prospectively collected.

Results: Among the 619 patients included in AKIKI, 462 received RRT and 459 were finally included, with 598 RRT catheters. Femoral site was chosen preferentially $(n=319,53 \%)$, followed by jugular $(n=256,43 \%)$ and subclavian $(n=23,4 \%)$. In multivariate analysis, continuous RRT modality was significantly associated with femoral site $(\mathrm{OR}=2.33$ (95\% Cl (1.34-4.07), $p=0.003$ ) and higher weight with jugular site [88.9 vs $83.2 \mathrm{~kg}, \mathrm{OR}=0.99(95 \% \mathrm{Cl} 0.98-1.00)$, $p=0.03]$. Investigator site was also significantly associated with the choice of insertion site $(p=0.03)$. Cumulative incidence of catheter replacement did not differ between jugular and femoral site [sHR 0.90 ( $95 \% \mathrm{Cl} 0.64-1.25)$, $p=0.67]$. Catheter dysfunction was the main reason for replacement $(n=47)$, followed by suspected infection $(n=29)$ which was actually seldom proven $(n=4)$. No mechanical complication (pneumothorax or hemothorax) occurred.
\end{abstract}

\footnotetext{
*Correspondence: stephanegaudry@gmail.com

†Didier Dreyfuss and Stéphane Gaudry contributed equally to this work

${ }^{31}$ Present Address: Service de Réanimation Médico-Chirurgicale, Hôpital

Avicenne, 125 Rue de Stalingrad, 93000 Bobigny, France

Full list of author information is available at the end of the article
}

\section{Springer Open}

(c) The Author(s) 2021. This article is licensed under a Creative Commons Attribution 4.0 International License, which permits use, sharing, adaptation, distribution and reproduction in any medium or format, as long as you give appropriate credit to the original author(s) and the source, provide a link to the Creative Commons licence, and indicate if changes were made. The images or other third party material in this article are included in the article's Creative Commons licence, unless indicated otherwise in a credit line to the material. If material is not included in the article's Creative Commons licence and your intended use is not permitted by statutory regulation or exceeds the permitted use, you will need to obtain permission directly from the copyright holder. To view a copy of this licence, visit http://creativeco mmons.org/licenses/by/4.0/. 
Conclusion: Femoral site was preferentially used in this prospective study of RRT catheters in 31 French intensive care units. The choice of insertion site depended on investigating center habits, weight, RRT modality. A high incidence of catheter infection suspicion led to undue replacement.

Keywords: Renal replacement therapy, Acute kidney injury, Vascular access, Catheter, Critical care

\section{Background}

The site of vascular access for acute renal replacement therapy (RRT) is a daily clinical question for intensivists and nephrologists taking care of patients with severe acute kidney injury (AKI).

Placement of temporary catheter at the subclavian site is not recommended because of the risk of vascular thrombosis or stenosis of the subclavian vein, which could hamper potential creation of arteriovenous fistula in these patients at risk of end-stage renal failure [1-8].

The CATHEDIA study, a major randomized controlled trial (RCT) comparing femoral and jugular sites for RRT catheter insertion, has shown similar rate of nosocomial event [catheter-tip colonization and catheter-related bloodstream infections (CRBI)] [9] and catheter dysfunction[10]. However, left jugular site was associated with higher rate of dysfunction; whereas, catheter-tip colonization was higher among patients with body mass index $(\mathrm{BMI})>28.4$ when femoral position was preferred.

These results, although in contradiction with some previous observational studies $[11,12]$ and recommendations $[13,14]$ that advised against femoral site, have led to consider both femoral and jugular sites acceptable. Recent guidelines recommend equally both sites (femoral and jugular) $[6,15]$ or favor right jugular site in others [16] given that there was a trend in favor of right jugular compared to femoral site regarding dysfunctions in the trial mentioned above.

Since the publication of this RCT and guidelines, there is a scarce of prospective observational data on the habits of intensivists concerning RRT catheter [17].

We published in 2016 a multicenter randomized controlled trial (AKIKI: Artificial Kidney Initiation in Kidney Injury) [18] on RRT timing initiation in intensive care units (ICU) patients with severe AKI (stage 3 of KDIGO classification). The AKIKI trial database provides several interesting prospective data regarding RRT catheters in 31 French ICUs.

We aimed to investigate RRT catheter site, duration of use, reason for catheter replacement, and complications, in particular infectious according to insertion site, in a large population of critically ill patients.

\section{Methods}

Study design and patients

We performed an ancillary study of the AKIKI trial, an open pragmatic RCT conducted in 31 ICUs in France from September 2013 through January 2016. Adults patients with severe AKI (stage 3 of KDIGO classification) and requirement for invasive mechanical ventilation and/or catecholamine infusion were randomly assigned (1:1) to either an early or a delayed RRT initiation strategy provided they had no life-threatening complication mandating immediate RRT. Detailed protocol is available elsewhere [18]. In the early strategy group, RRT was initiated as soon as possible after randomization. In the delayed group, RRT was initiated only in the context of severe metabolic or clinical abnormalities.

All patients who underwent at least one RRT session were enrolled in the present study.

The original trial was approved by the ethical committee of the French Society of Intensive Care Medicine and by the competent French legal authority (Comité de Protection des Personnes d'Ile de France VI, ID RCB 2013A00765-40, NCT01932190) for all participating centers. Patients or their surrogates were informed both verbally and with a written document by the investigators. They could refuse to participate at any time, and their decision was recorded in patient files.

\section{Catheter management}

As a pragmatic study, choice of catheter insertion site and management were left at the discretion of each study center and investigator. However, investigators were encouraged to follow the current national guidelines [6], i.e., equivalence of right internal jugular and femoral site in terms of risk for dysfunction or infection, preference for internal jugular site for patients with a body mass index above $28 \mathrm{~kg} / \mathrm{m}^{2}$ to reduce infectious risk. Guidelines also recommend using catheters of diameter $>12 \mathrm{~F}$ and length $\geq 24 \mathrm{~cm}$ for femoral site and ultrasound guidance for jugular and femoral vein catheter placement. Removal of RRT catheter was mandated as soon as it is no longer necessary. Information on catheter tip culture at removal, skin cleaning protocols and RRT catheter lock solutions used in participating centers are provided in the Additional file 1: Table S1. 


\section{Endpoints}

We sought to describe the sites of RRT catheter insertion, and factors potentially involved in the choice between femoral and jugular for the first catheter (age, weight, sex, SAPS3 and SOFA, acute respiratory distress syndrome (ARDS), invasive mechanical ventilation, hemorrhagic risk (defined as $\geq$ one of the following criteria: platelets $<100 \mathrm{G} / \mathrm{L}$, prothrombin ratio $<50 \%$, anticoagulation therapy), peripheral vascular disease, RRT modality, investigator site).

We also assessed duration of the first catheter use and reason for replacement (catheter dysfunction, thrombosis, suspicion of infection, proven infection or others, as defined as requiring catheter replacement according to the investigator) according to the insertion site.

Finally, we analyzed complications related to all RRT catheters: pneumothorax requiring exsufflation or drainage, hemothorax, gas embolism, bleeding requiring transfusion or hemostatic procedure, arterio-venous fistula, symptomatic deep venous thrombosis (confirmed with Doppler or CT angiography), bacteremia and fungemia. For RRT catheter associated bloodstream infections, definitions from IDSA 2009 guidelines were used (see Additional file 2: Table S2 [19]).

All data were prospectively collected during the AKIKI trial.

\section{Statistical methods}

Data were expressed as number (percent) for qualitative variables and mean (SD) for quantitative variables. Between groups, Pearson's Chi-squared tests or Fisher's exact tests as appropriate were used to compare qualitative variables and Wilcoxon rank sum tests for quantitative variables. To assess the factors potentially involved in the choice between femoral and jugular for the first catheter, a mixed logistic regression model was used. The fixed effects were: randomization arm, age, sex, weight, SAPS3, ARDS, invasive mechanical ventilation, hemorrhagic risk, peripheral vascular disease and the first RRT modality. The investigator study center variable was introduced as random effect. This center effect was evaluated by estimating the corresponding random effect variance component with its $95 \%$ confidence interval [20]. We also estimated the intra-class correlation (ICC) interpreted as the percentage of variance explained by the center effect. Collinearity was assessed using the variance inflation factor (VIF) which was estimated for each fixed predictor. A VIF lower than 1.5 was judged adequate.

Kaplan-Meier survival curves were used to describe duration of catheter's use. To assess the impact of the choice between femoral and jugular for the first catheter on subsequent catheter replacement requirement, we used Fine and Gray competing risk survival model weighted on inverse probability of treatment weights (IPTW). A multivariable logistic regression model was performed to estimate a propensity score for each patient using all covariates described above. To assess weighting balanced measured covariates between the two groups, we used the weighted standardized mean difference, and we considered an absolute standardized difference less than 0.1 as evidence of balance [21]. In the second step, a Fine and Gray model [22], weighted on IPTW, was fitted to take into account the risk of death. The event of interest was the catheter replacement and the competing event was death. Time was defined as delay between dialysis initiation and catheter replacement or death for patients who had one of these events, or duration of catheter without replacement or complication (censored data). Cumulative incidence and subdistribution hazard ratio (sHR) were estimated with their 95\% confidence intervals.

All analyses were performed at a two-sided $\alpha$ level of $5 \%$ and conducted with $\mathrm{R}$ software version 3.3.3 (R Foundation for Statistical Computing).

\section{Results}

\section{Patient population}

Among the 619 patients included in the AKIKI study, 462 received RRT at least one time. Three were excluded due to missing data, leaving 459 patients in the present study (Fig. 1). A total of 598 catheters were included in the analysis (3 were excluded due to missing data).

patient characteristics are detailed in Table 1.

\section{Choice of RRT catheter insertion site}

Table 2 shows the number of RRT catheters according to the insertion site. Femoral site was chosen in $53 \%(n=319)$ of cases, jugular site in $43 \%(n=256)$. As expected, the choice of subclavian site was very rare (less than $5 \%, n=23)$. For the first catheter, among the 439 catheters placed in jugular or femoral site (considering that for the subclavian placement, jugular or femoral was not an option), the sample proportion of 248/439 femoral placement (56.5\%, 95\% CI 51.7-61.2) was statistically significantly different from a $50 \%$ expected proportion with $p=0.008$ ( $z$ test to compare a single proportion to population estimate).

In multivariate analysis (Table 3), continuous RRT modality was significantly associated with preference of femoral site as the first choice $[\mathrm{OR}=2.22$ (95\% CI 1.28-3.86), $p=0.005]$. On the opposite, higher weight was associated with preference for jugular site [OR $=0.99$ (95\% CI 0.98-0.99), $p=0.03$ ]. Investigator site was also significantly associated with the choice of insertion site (between femoral and jugular) in multivariate analysis 


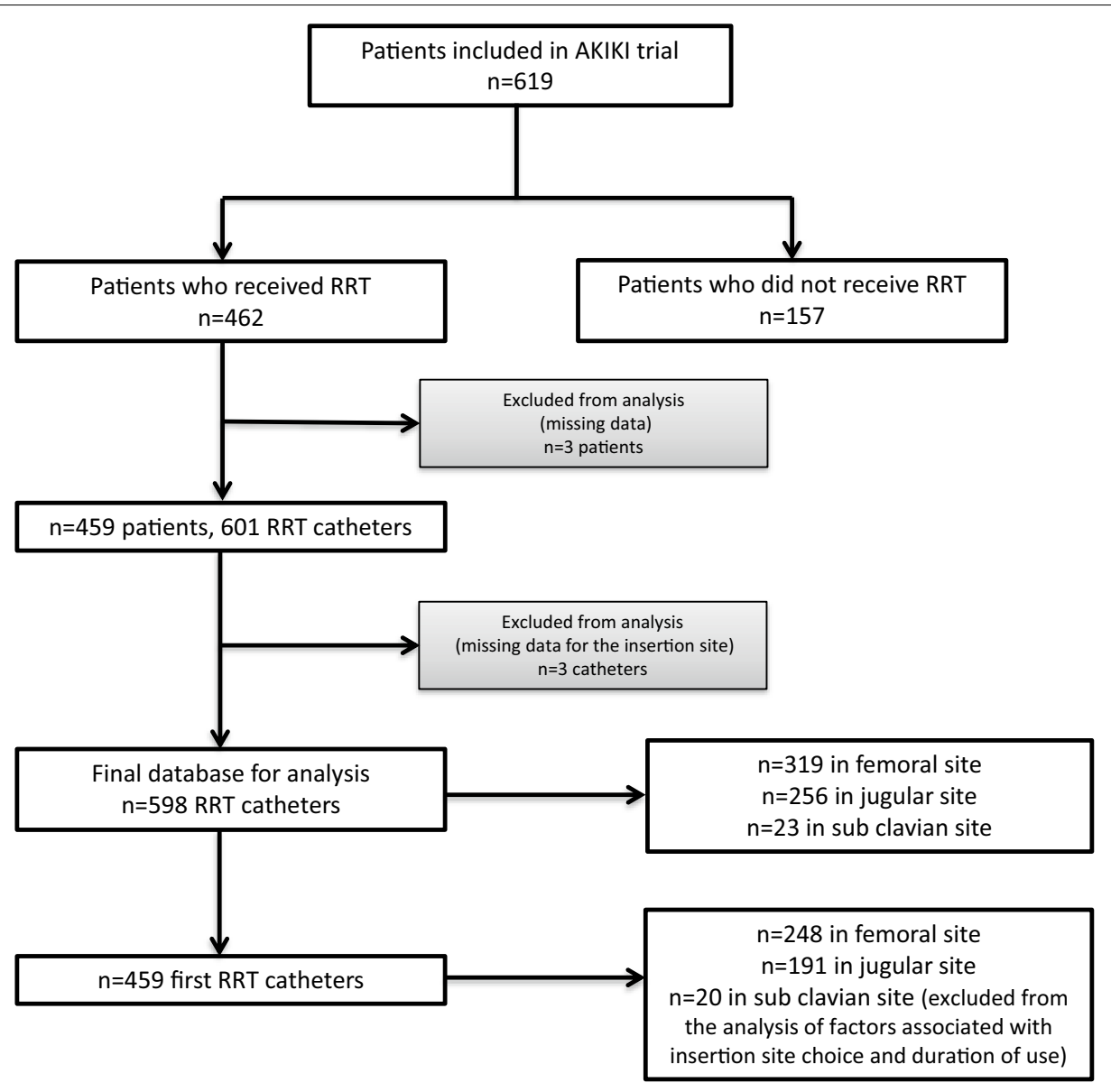

Fig. 1 Flow chart

$(p=0.03)$. The percentage of variance explained by the investigator center was of $10.5 \%$.

\section{Duration of use and reasons for catheter replacement}

Overall median duration of catheter use was 5 days for both femoral (IQR 2-8), and jugular (IQR 3-8) catheters (log rank test $p=0.84$, Fig. 2).

In a Fine and Gray competing risk model analysis weighted on inverse probability weighting treatment with death as competing event (Additional file 4: Table S4, Additional file 6: Figure S2), probability of first catheter replacement did not differ between femoral and jugular site $[\mathrm{sHR}=0.90$ (95\% CI 0.64-1.25), $p=0.67$, Additional file 5: Figure S1].

Cumulative incidence of first catheter replacement at day 7 were, respectively, 0.2 (95\% CI 0.14-0.26) for femoral and 0.19 (95\% CI 0.12-0.25) for jugular site $(p=0.77$, Table 4).

Reasons for first catheter replacement (Table 5) were not different for femoral and jugular sites. The main reason was dysfunction [26/49 for femoral (53.1\%), 16/36 for jugular (44.4\%)]. All sub-clavian catheter replacements resulted from dysfunction. The absolute rates of replacement for dysfunction among all catheters were $10.4 \%$ $(26 / 248)$ for femoral and $8.4 \%(16 / 191)$ for jugular site.

Of note, suspected infection led to 14 (28.6\%) replacements in femoral group and 15 (41.7\%) in jugular group; whereas, infection leading to replacement was documented in only 1 and 3 instances for femoral and jugular sites, respectively.

\section{Catheter-related complications}

Catheter-related complications according to insertion site are detailed in Table 6.

Rates of catheter-related bloodstream infections (bacteremia or fongemia) were rare and not different between femoral and jugular sites (respectively, 3.1\%o and 4.4\%o catheter-days). Rates of catheter-related infections according to randomization arm (i.e., RRT initiation strategy) are provided in the Additional file 7: Table S7.

No pneumothorax nor hemothorax directly related to RRT catheter insertion occurred during the study. One 
Table 1 Characteristics of the patients at baseline

\begin{tabular}{|c|c|}
\hline Characteristics & Patients $(n=459)$ \\
\hline Sex (men/women) $n(\%)$ & $310 / 149(67.5 / 32.5)$ \\
\hline Age (year) $( \pm S D)$ & $65.6( \pm 13.7)$ \\
\hline Weight $( \pm S D)$ & $85.5( \pm 22.2)$ \\
\hline Chronic renal failure $\mathrm{n}(\%)$ & $43(9)$ \\
\hline Congestive heart failure $n(\%)$ & $41(9)$ \\
\hline Ischemic heart disease $n(\%)$ & $43(9)$ \\
\hline Hypertension $n(\%)$ & $235(51)$ \\
\hline Diabetes mellitus $n(\%)$ & $121(26)$ \\
\hline Invasive mechanical ventilation $n(\%)$ & $394(86)$ \\
\hline $\operatorname{ARDS}^{\mathrm{b}} n(\%)$ & $156 / 456(34)$ \\
\hline SAPS $\| I^{c}( \pm S D)$ & $73.4( \pm 14.0)$ \\
\hline $\operatorname{SOFA}^{\mathrm{d}}( \pm \mathrm{SD})$ & $11.2( \pm 3.0)$ \\
\hline Central venous catheter $n(\%)$ & $411(90)$ \\
\hline
\end{tabular}

All data at enrollment. Plus-minus values are means \pm SD (standard deviation)

a Chronic renal failure defined as eGFR $<60 \mathrm{ml} / \mathrm{mn}$

${ }^{b}$ ARDS: Berlin definition

c The Simplified Acute Physiology Score (SAPS) III ranges from 0 to 146, with higher scores indicating more severe disease and a higher risk of death

d The Sepsis-related Organ Failure Assessment (SOFA) score ranges from 0 to 24, with higher scores indicating more severe organ failure

Table 2 Number of catheters according to insertion site

\begin{tabular}{lllll}
\hline Insertion site & Total & First & Second & Third and $\geq$ \\
\hline Femoral & $319(53 \%)$ & $248(54 \%)$ & $49(52 \%)$ & $22(43 \%)$ \\
Jugular & $256(43 \%)$ & $191(42 \%)$ & $44(46 \%)$ & $21(53 \%)$ \\
Sub-clavian & $23(4 \%)$ & $20(4 \%)$ & $2(2 \%)$ & $1(3 \%)$ \\
Total & $598(100 \%)$ & 459 & 95 & 44 \\
\hline
\end{tabular}

catheter-related gas embolism and 1 catheter-related fistula occurred in the femoral site. Rate of catheter-related bleeding requiring transfusion or hemostatic procedure was very rare and similar in both sites [2 for femoral $(0.6 \%)$ and 2 for jugular $(0.8 \%)$ ]. Finally, we observed very few symptomatic deep venous thromboses ( 2 among femoral catheters, and 3 among jugular ones).

\section{Discussion}

In this prospective study of RRT catheters among critically ill patients with severe acute kidney injury in 31 French ICUs, femoral site was preferentially used, before jugular site. The choice of insertion site depended on RRT modality (femoral site was more frequently chosen for continuous modality), patient weight (jugular site was preferred for higher weight patients) and investigator study center habits. The rate of dysfunction and complications did not differ between jugular and femoral catheters. Suspicion of infection led to replacement of many catheters but was actually seldom proven, and the incidence of clinically significant infection was quite low.

The main objective of this study was to show a «reallife» use of RRT catheters in a large population of ICU patients. We took advantage of our recently published large multicenter randomized controlled trial (AKIKI) involving severely ill patients (acute kidney injury stage 3 and mostly receiving mechanical ventilation and catecholamine infusion), although one should note that patients with life-threatening complications of severe AKI such as severe hyperkalemia were excluded from the trial, thus limiting generalizability of our findings in this population. Few years after the publication of an important RCT on the topic of RRT catheters [9, 10], the present study provides an interesting snapshot of RRT catheter use in the ICU in France. Furthermore, one should note that our study population included more severe patients than the aforementioned trial (85\% receiving catecholamines vs 35-40\%), thus providing interesting data in this particular population.

The small difference regarding the choice of the insertion site (between femoral and jugular) is probably the consequence of the guidelines $[6,15]$ leaving the choice between the 2 sites. These guidelines are based on the results of the CATHEDIA study [9, 10, 23]. This French multicenter RCT published in 2008 included 736 dialyzed ICU patients and found no difference in terms of infectious complications (colonizations and bloodstream infections) [9] nor in dysfunctions of catheters [10] between these two sites. Choice of the site of insertion for the second catheters, which were probably placed in less urgent situations, were similar. These findings suggest that the conclusions of this trial have been adopted by clinicians, who do not hesitate to use femoral site.

Of note, subclavian site was the choice for $4 \%$ of the first catheters used. This site has been prohibited in every nephrology recommendation for many years, because of the important risk of thrombosis or stenosis of the subclavian vein. Indeed, such a complication hampers a potential creation of arteriovenous fistula, a major issue in patients with AKI who are now considered to be at risk of end stage renal failure [1-8].

Higher weight was associated with preference for jugular site insertion, in agreement with the demonstration of a significantly lower incidence of colonization in the jugular compared to femoral site by the CATHEDIA study sub-group analysis [9] in patients with a BMI > 28.4. Noteworthy, however, is the fact that we did not record BMI. To our knowledge, our study is the first to show that RRT modality impacts the choice of RRT catheter site, continuous RRT (CRRT) being associated with more femoral catheterization. This finding fits with the higher proportion of femoral RRT catheters (67\%) used in the 
Table 3 Factors potentially associated with the choice of insertion site in univariate and multivariate analyses (between femoral and jugular for the first catheter, $n=439$ )

\begin{tabular}{|c|c|c|c|c|c|}
\hline \multirow[t]{2}{*}{ Variable ${ }^{f}$} & \multirow[t]{2}{*}{ Femoral $(n=248)$} & \multirow[t]{2}{*}{ Jugular $(n=191)$} & \multirow{2}{*}{$\begin{array}{l}\text { Univariate analysis } \\
p \text { values }^{\mathrm{a}}\end{array}$} & \multicolumn{2}{|c|}{ Multivariate analysis } \\
\hline & & & & OR (Cl 95) & $p$ values ${ }^{b}$ \\
\hline Randomization arm:delayed group & $81(32.7 \%)$ & $68(35.6 \%)$ & 0.52 & $0.97(0.59-1.58)$ & 0.89 \\
\hline Age-years & $64.51(14.62)$ & $66.21(12.54)$ & 0.44 & & \\
\hline Age $>70$ years & $103(41.5 \%)$ & $77(40.3 \%)$ & 0.80 & $1.08(0.66-1.76)$ & 0.78 \\
\hline Sex (Male) & $174(70.2 \%)$ & $122(63.9 \%)$ & 0.16 & $1.43(0.89-2.37)$ & 0.16 \\
\hline Weight at randomization — mean (SD) & $83.21(19.9)$ & $88.92(25.2)$ & 0.07 & $0.99(0.98-0.99)$ & 0.03 \\
\hline Peripheral vascular disease & $35(14.1 \%)$ & $21(11 \%)$ & 0.33 & $1.46(0.70-3.07)$ & 0.32 \\
\hline ARDS at randomization & $88 / 246(35.8 \%)$ & $61 / 190(32.1 \%)$ & 0.42 & $1.00(0.59-1.68)$ & 0.99 \\
\hline SAPS 3 score at randomization - mean (SD) & $73.6(15.5)$ & $73.5(12.5)$ & 0.81 & $1.00(0.98-1.01)$ & 0.58 \\
\hline Invasive mechanical ventilation & $211(85.1 \%)$ & $164(85.9 \%)$ & 0.82 & $0.85(0.41-1.73)$ & 0.38 \\
\hline Hemorrhagic risk ${ }^{c}$ & $172 / 225(76.4 \%)$ & $124 / 166(74.7 \%)$ & 0.69 & $1.01(0.59-1.72)$ & 0.98 \\
\hline Modality of first RRT Continuous & $125(50.6 \%)$ & 75 (39.3\%) & 0.02 & $2.22(1.28-3.86)$ & 0.005 \\
\hline Investigator center ${ }^{d}$ & - & - & - & - & $0.03^{e}$ \\
\hline
\end{tabular}

a $p$ values: Pearson's Chi-squared for qualitative data and Wilcoxon rank sum test for quantitative data test.

${ }^{b} p$ values: associated to multivariate logistic regression model.

c Hemorrhagic risk: $\geq$ one of the following criteria: platelets $<100 \mathrm{G} / \mathrm{L}$, prothrombin ratio $<50 \%$, anticoagulation therapy.

d Investigator center is a variable with 32 levels.

e $p$ value associated with investigator center: This center effect was evaluated by estimating the corresponding random effect variance component. The variance of center variable was of $0.38(95 \% \mathrm{Cl}, 0.28-0.89)$. We also estimated the percentage of variance explained by the effect center and it was of $10.5 \%$

f Collinearity control: variance inflation factor (VIF) was lower than 1.5, thus judged adequate, for each fixed predictor (Additional file 3: Table S3).

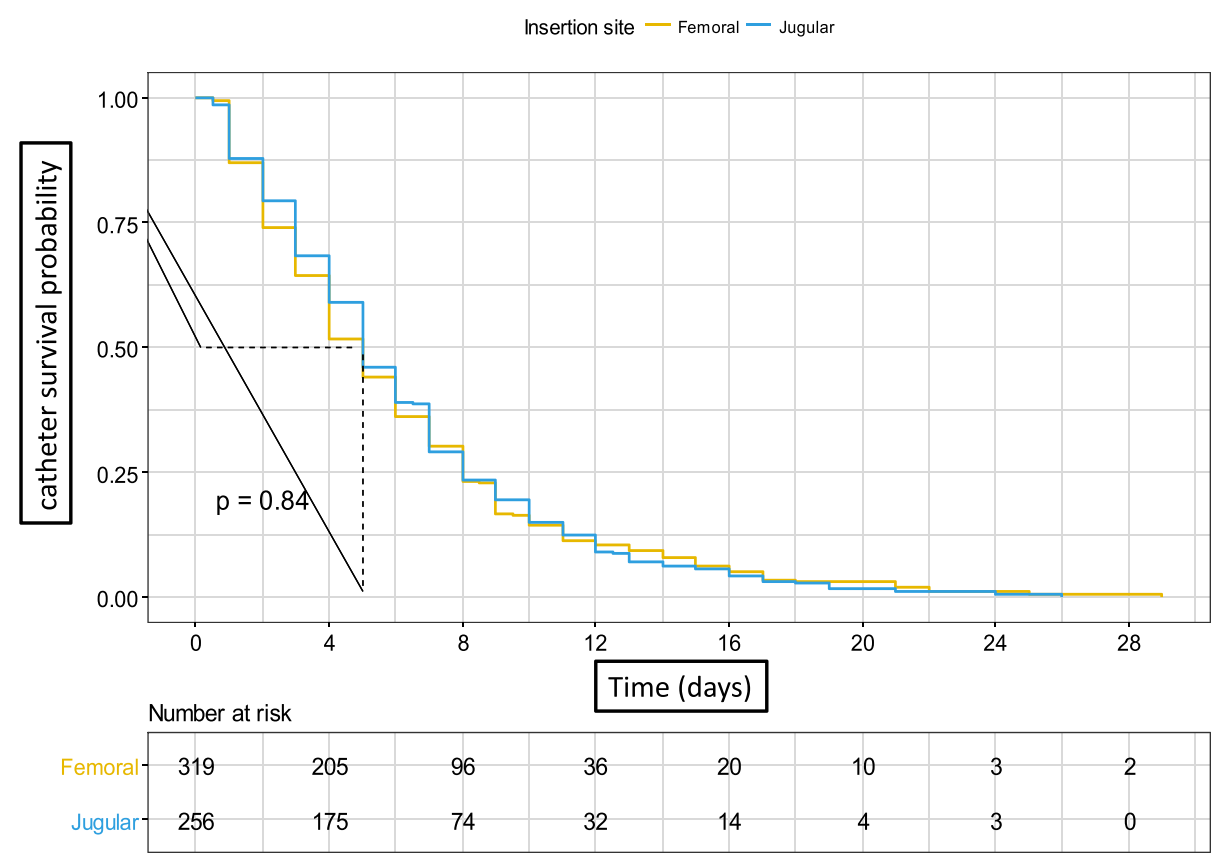

Fig. 2 Duration of catheter use. Legend: Median duration of use was 5 days for both femoral (IQR 2-8), and jugular (IQR 3-8) catheters ( $p=0.84)$ 
Table 4 Cumulative incidence of catheter replacement and death according to insertion site (first catheter, jugular or femoral)

\begin{tabular}{lllll}
\hline Event & Site & \multicolumn{2}{l}{ Cumulative incidence (CI 95) } & Day 21 \\
\cline { 3 - 4 } & & Day 7 & Day 14 & $0.41(0.29-0.53)$ \\
\hline Catheter replacement & Femoral & $0.2(0.14-0.26)$ & $0.29(0.2-0.37)$ & $0.37(0.25-0.48)$ \\
& Jugular & $0.19(0.12-0.25)$ & $0.34(0.24-0.45)$ & $0.39(0.29-0.5)$ \\
Death & Femoral & $0.23(0.17-0.29)$ & $0.36(0.27-0.45)$ & $0.35(0.22-0.48)$ \\
\hline
\end{tabular}

Table 5 Reasons for first catheter replacement according to insertion site

\begin{tabular}{lllll}
\hline Reason for catheter replacement & Total & Femoral & Jugular & Sub-clavian \\
\hline Dysfunction & $47(52.2 \%)$ & $26(53.1 \%)$ & $16(44.4 \%)$ & $5(100 \%)$ \\
Thrombosis & $1(1.1 \%)$ & $1(2 \%)$ & $0(0 \%)$ & $0(0 \%)$ \\
Catheter infection suspicion & $29(32.2 \%)$ & $14(28.6 \%)$ & $15(41.7 \%)$ & $0(0 \%)$ \\
Proven catheter infection & $4(4.4 \%)$ & $1(2 \%)$ & $3(8.3 \%)$ & $0(0 \%)$ \\
Other & $9(10 \%)$ & $7(14.3 \%)$ & $2(5.6 \%)$ & $0(0 \%)$ \\
Total & 90 & 49 & 36 & 5 \\
\hline
\end{tabular}

Table 6 Possible catheter-related complications according to insertion site (all catheters)

\begin{tabular}{|c|c|c|c|c|}
\hline & $\begin{array}{l}\text { Femoral }(n=319 \\
\text { catheters) }\end{array}$ & $\begin{array}{l}\text { Jugular }(n=256 \\
\text { catheters) }\end{array}$ & $\begin{array}{l}\text { Sub-clavian ( } n=23 \\
\text { catheters) }\end{array}$ & $\begin{array}{l}p \text { values } \\
\text { (femoral vs } \\
\text { jugular) }\end{array}$ \\
\hline \multicolumn{5}{|l|}{ Infectious complications } \\
\hline \multicolumn{5}{|l|}{ Catheter-related bloodstream infections } \\
\hline Number $^{a}$ & $6(1.9 \%)$ & $7(2.7 \%)$ & 0 & 0.58 \\
\hline Incidence rate $(\%)^{\mathrm{b}}$ & $2.2 \%$ & $3.2 \%$ & & - \\
\hline Incidence density (\%o catheter-days) ${ }^{c}$ & $3.1 \%$ & $4.4 \% 0$ & & - \\
\hline Bloodstream infections without cause $\mathrm{e}^{\mathrm{a}}$ & $5(1.6 \%)$ & $1(0.4 \%)$ & 0 & 0.23 \\
\hline \multicolumn{5}{|l|}{ Mechanical complications ${ }^{\mathrm{a}}$} \\
\hline Pneumothorax & 0 & 0 & 0 & 1.00 \\
\hline Hemothorax & 0 & 0 & 0 & 1.00 \\
\hline Gas embolism & $1(0.3 \%)$ & 0 & 0 & 1.00 \\
\hline Fistula & $1(0.3 \%)$ & 0 & 0 & 1.00 \\
\hline $\begin{array}{l}\text { Bleeding requiring transfusion or hemostatic } \\
\text { procedure }\end{array}$ & $2(0.6 \%)$ & $2(0.8 \%)$ & 0 & 1.00 \\
\hline \multicolumn{5}{|l|}{ Thrombotic complications ${ }^{\mathrm{a}}$} \\
\hline Symptomatic deep venous thrombosis ${ }^{a}$ & $2(0.6 \%)$ & $3(1.2 \%)$ & 0 & 0.66 \\
\hline
\end{tabular}

All complications above are «catheter related» except «bloodstream infections without cause»

$P$ values: Fisher's exact test for count data. No $p$ value calculated for incidence rate and incidence density because the two samples were not independent. Patients could have a femoral and jugular catheter

a All percentages are expressed as number of events per number of catheters except for incidence rate and incidence density

b Incidence rate: number of patients contracting an infection per number of patients at risk (272 patients under femoral catheter and 221 patients under jugular catheter)

c Incidence density: number of infection per 1000 catheter-days

RENAL study [24] compared to our trial, as it assessed optimal dialysis dose among CRRT-treated patients only. One should hypothesize that patients receiving intermittent RRT modality are more susceptible to be mobilized (sitting position) between the sessions. In this condition, some intensivists may avoid femoral site due to the risk of femoral thrombosis. 
Quite surprisingly, hemorrhagic risk (defined as at least one of the following criteria: platelets $<100 \mathrm{G} / \mathrm{L}$, prothrombin ratio $<50 \%$, curative anticoagulation therapy) was not associated with insertion site, unlike results from a secondary analysis of the ATN trial [25], that showed a tendency to place more femoral catheters in coagulopathic patients. It should be mentioned that the use of antiplatelets agents was not recorded in our study though.

We acknowledge that central venous infusion catheters site (femoral or jugular) was not known at randomization in the AKIKI trial. This could be an important factor involved in the choice of RRT catheter insertion site, given that $90 \%$ of patients had central venous lines at baseline.

The first reason for first catheter replacement was dysfunction (approximately half of cases) in both jugular and femoral site. Rate of dysfunction leading to catheter replacement among first catheters (around 10\%) was similar to that previously reported in the literature, notably in the CATHEDIA study [10]. Unfortunately, we were not able to provide the information of left versus right jugular insertion. One should know that in the CATHEDIA trial, dysfunctions were more frequent in left jugular site than in right jugular and femoral sites.

We found a high incidence (around 30\%) of catheter replacement related to infection suspicions; whereas, only a few infections were actually proven (around $4 \%$ of catheter replacements). This underlines the lack of reliable diagnostic technique for catheter-related infections $[26,27]$. These unnecessary changes of catheters have negative consequences such as waste of time and inherent risks of new catheter insertion.

Only 6 (3.1\%o catheter-days) catheter-related bloodstream infections in femoral and 7 (4.4\%o catheter-days) in jugular site were found. These are rather low rates (even if we aggregate the number of catheter-related bloodstream infections with those of unknown origin) in this population of very severe and fragile patients, especially given the fact that RRT catheters might be associated with more CRBIs than other central catheters [28]. These results, similar to those from recent studies $[29,30]$, reflect the progress achieved during the last years regarding infection prevention and catheter care [31,32], due to generalization of infectioncontrol «bundles», mainly sterile precautions at the time of catheter insertion and at each manipulation $[6$, 16, 33-36]. The short duration of catheter use in our study (median 5 days) also highlights the importance of prompt removal of unnecessary catheters. Even more importantly, it seems obvious that the best way to prevent catheter-related infection is to avoid unnecessary catheter insertion. This is particularly true in that study which stems from an RCT showing the inutility of $50 \%$ of RRT initiation: indeed, there were twice as many patients with catheter-related bloodstream infection in the early initiation arm (10\%) compared to the delayed strategy (5\%) of the AKIKI trial [18].

No mechanical complication (pneumothorax and hemothorax) related to RRT catheters occurred, among our 459 patients, 598 catheters (including 256 jugular and 20 subclavian insertion sites). This may be due to the generalization of ultrasound guidance for catheter placement [37] which was strongly recommended at the time of AKIKI $(6,16,38-40)$ for jugular site and for femoral site although we did not record the proportion of ultrasound guidance in the present study.

Our study has potential limitations, mostly driven by its ancillary design, within a RCT not directly aiming at investigating RRT catheters. First, proportions of left and right jugular catheters were not collected. Second, use of echography to guide catheter insertion was not recorded. Third, position (femoral or jugular) of central venous catheters already in place for fluid and drug administration at randomization was not known. This could have influenced the choice of RRT catheter insertion site. Fourth, the definitions of catheter dysfunction, thrombosis and suspicion of infection were not prespecified during the AKIKI trial but left to the appreciation of investigators. Fifth, duration or intervals between RRT sessions and clinical surveillance of insertion sites were left at discretion of the physicians, which might have influenced rate and time to catheter replacement. Of note, analyses regarding exposures associated with insertion site, rate and time to catheter replacement were limited to first catheter per patient, limiting generalizability of our findings to this situation.

\section{Conclusion}

Femoral site was preferentially used in this observational study of RRT catheters in 31 French intensive care units. The choice of insertion site depended on RRT modality, patient weight and investigating center habits. There were similar rates of dysfunctions and complications between femoral and jugular sites.

We found a high incidence of undue catheter replacement related to infection suspicion, whereas only a few proven catheter infections actually occurred. Therefore, one interesting research focus could be trying to develop more accurate diagnosis techniques of catheter related infections to avoid undue RRT catheter changes. Unnecessary RRT should be avoided to minimize catheter-related complications. 


\section{Abbreviations}

RRT: Renal Replacement Therapy; AKI: Acute Kidney Injury; RCT: Randomized Controlled Trial; ICU: Intensive Care Unit; AKIKI: Artificial Kidney Initiation in Kidney Injury; CRBI: Catheter Related Bloodstream Infections; BMI: Body Mass Index; KDIGO: Kidney Disease Improving Global Outcomes; SAPS: Simplified Acute Physiology Score; SOFA: Sepsis-related Organ Failure Assessment; ARDS: Acute Respiratory Distress Syndrome.

\section{Supplementary Information}

The online version contains supplementary material available at https://doi. org/10.1186/s13613-021-00843-3.

Additional file 1: Table S1. Catheter tip culture at removal, skin cleaning protocol and catheter lock solution according to participating centers.

Additional file 2: Table S2. Catheter-related bacteremia or fungemia definitions (from Guidelines IDSA 2009, CID 2009 Dec 1 ;49:1-45).

Additional file 3: Table S3. Collinearity control: variance inflation factors for fixed effects factors potentially involved in the choice between femoral and jugular for the first catheter insertion.

Additional file 4: Table S4. Covariate balance between femoral and jugular groups before and after propensity score weighting for first catheter insertion.

Additional file 5: Figure S1. Cumulative incidence of catheter replacement and death, for first catheter, according to insertion site (jugular and femoral).

Additional file 6: Figure S2. Propensity score distribution between femoral and jugular groups for first catheter insertion.

Additional file 7: Table S5. Possible infectious catheter-related complications according to insertion site and arm of randomization (among patients who underwent RRT and catheter insertion).

\section{Acknowledgements}

We are grateful to all healthcare professionals, physicians, and nurses who participated in the AKIKI trial.

\section{Authors' contributions}

NB, SG and DD wrote and reviewed the manuscript. SL and DH performed statistical analysis. Each author contributed important intellectual content during manuscript drafting or revision and accepts accountability for the overall work by ensuring that questions pertaining to the accuracy or integrity of any portion of the work are appropriately investigated and resolved.

\section{Funding}

The original AKIKI trial was supported by a Grant from the Programme Hospitalier de Recherche Clinique National, 2012 (AOM12456), funded by the French Ministry of Health.

\section{Availability of data and materials}

The datasets used during the current study are available from the corresponding author on reasonable request.

\section{Declarations}

\section{Ethics approval and consent to participate}

The original trial was approved by the ethical committee of the French Society of Intensive Care Medicine and by the competent French legal authority (Comité de Protection des Personnes d'lle de France VI, ID RCB 2013-A0076540, NCT01932190) for all participating centers. Patients or their surrogates were informed both verbally and with a written document by the investigators. They could refuse to participate at any time, and their decision was recorded in patient files.

\section{Consent for publication}

Patients or their surrogates were informed both verbally and with a written document by the investigators. They could refuse to participate at any time, and their decision was recorded in patient files.

\section{Competing interests}

None of the authors have competing interests in the manuscript.

\section{Author details}

${ }^{1}$ AP-HP, Hôpital Européen Georges Pompidou, Service de Néphrologie, 75015 Paris, France. ${ }^{2}$ Département de Biostatistiques, Santé Publique Et Information Médicale, AP-HP, Hôpital Pitié-Salpêtrière, 75013 Paris, France. ${ }^{3}$ INSERM, ECEVE, U1123, CIC 1421, F-75013 Paris, France. ${ }^{4}$ Faculté de Médecine Sorbonne, Sorbonne Université, Université, Paris, France. ${ }^{5}$ Réanimation Médico-Chirurgicale, CHG, La Roche-sur-Yon, France. ${ }^{6}$ Service de Réanimation, CHU de Pointe À Pitre-Abymes, CHU de La Guadeloupe, Pointe-à-Pitre, France. ${ }^{7}$ Réanimation Polyvalente, CH René Dubos, 95301 Pontoise, France. ${ }^{8}$ Réanimation Médicale CHU Bordeaux, Hôpital Pellegrin, 33000 Bordeaux, France. ${ }^{9}$ Service de Réanimation, Centre Hospitalier Sud Francilien, Corbeil Essonne, France. ${ }^{10}$ Département de Réanimation Médicale Et Médecine Hyperbare, CHU Angers, Université D'Angers, Angers, France. ${ }^{11}$ Réanimation Médicale, CHU Rouen, 76000 Rouen, France. ${ }^{12}$ Assistance Publique-Hôpitaux de Paris, Hôpitaux Universitaires Henri Mondor, DHU A-TVB, Service de Réanimation Médicale, Créteil, France. ${ }^{13}$ CARMAS Research Group and UPEC-Université Paris-Est Créteil Val de Marne, Créteil, France. ${ }^{14}$ Réanimation Médicale, Hôpital Gabriel Montpied, CHU de Clermont-Ferrand, Clermont-Ferrand, France.

${ }^{15}$ Réanimation Médico-Chirurgicale, Hôpital de La Source, Centre Hospitalier Régional D'Orléans, BP 6709, 45067 Orléans Cedex, France. ${ }^{16}$ Service de Pneumologie Et Réanimation Médicale, APHP, Groupe Hospitalier PitiéSalpêtrière, Paris, France. ${ }^{17}$ Centre de Réanimation, $\mathrm{CHU}$ de Lille, Faculté de Médecine, Université de Lille, 59000 Lille, France. ${ }^{18}$ Réanimation Médicale Et Toxicologique, Hôpital Lariboisière, INSERM U1144, Université Paris-Diderot, Paris, France. ${ }^{19}$ Réanimation Polyvalente, CH Victor Dupouy, 95107 Argenteuil Cedex, France. ${ }^{20}$ Service de Réanimation Des Détresses Respiratoires Aiguës Et Infections Sévères, Hôpital Nord Marseille, 13015 Marseille, France. ${ }^{21}$ Service de Réanimation Médicale INSERM U1088, Centre Hospitalier Universitaire de Picardie, Amiens, France. ${ }^{22}$ Réanimation Médicale, Hôpital de La Croix Rousse, 69004 Lyon, France. ${ }^{23}$ Réanimation, CH Cholet, 49300 Cholet, France. ${ }^{24}$ Centre Hospitalier Inter-Communal, Service de Réanimation Polyvalente Adulte, Créteil, France. ${ }^{25}$ Univ Pierre Et Marie Curie, Sorbonne Universités, 75013 Paris, France. ${ }^{26}$ Univ Paris Diderot, Sorbonne Paris Cité, IAME, UMRS 1137 , 75018 Paris, France. ${ }^{27}$ AP-HP, Service de Réanimation Médico-Chirurgicale, Hôpital Louis Mourier, 92700 Colombes, France. ${ }^{28}$ Université de Paris, Paris, France. ${ }^{29}$ French National Institute of Health and Medical Research (INSERM), UMR_S1155, Remodeling and Repair of Renal Tissue, Hôpital Tenon, Sorbonne Université, 75020 Paris, France. ${ }^{30}$ AP-HP, Service de Réanimation Médico-Chirurgicale, Hôpital Avicenne, 93008 Bobigny, France. ${ }^{31}$ Present Address: Service de Réanimation Médico-Chirurgicale, Hôpital Avicenne, 125 Rue de Stalingrad, 93000 Bobigny, France.

Received: 5 January 2021 Accepted: 24 March 2021 Published online: 08 April 2021

\section{References}

1. Hernández D, Díaz F, Rufino M, Lorenzo V, Pérez T, Rodríguez A, et al. Subclavian vascular access stenosis in dialysis patients: natural history and risk factors. J Am Soc Nephrol JASN août. 1998;9(1):1507-10.

2. Trerotola SO, Kuhn-Fulton J, Johnson MS, Shah H, Ambrosius WT, Kneebone PH. Tunneled infusion catheters: increased incidence of symptomatic venous thrombosis after subclavian versus internal jugular venous access. Radiology. 2000;217(2):89-93.

3. Bambauer R, Inniger R, Pirrung KJ, Schiel R, Dahlem R. Complications and side effects associated with large-bore catheters in the subclavian and internal jugular veins. Artif Organs avr. 1994;18(3):318-21.

4. Coca SG, Singanamala S, Parikh CR. Chronic kidney disease after acute kidney injury: a systematic review and meta-analysis. Kidney Int mars. 2012;81(4):442-8.

5. Rimes-Stigare C, Frumento P, Bottai M, Mårtensson J, Martling C-R, Walther SM, et al. Evolution of chronic renal impairment and long-term 
mortality after de novo acute kidney injury in the critically ill; a Swedish multi-centre cohort study. Crit Care Lond Engl. 2015;19(5):221.

6. Vinsonneau C, Allain-Launay E, Blayau C, Darmon M, Ducheyron D, Gaillot $T$, et al. Renal replacement therapy in adult and pediatric intensive care: recommendations by an expert panel from the French Intensive Care Society (SRLF) with the French Society of Anesthesia Intensive Care (SFAR) French Group for Pediatric Intensive Care Emergencies (GFRUP) the French Dialysis Society (SFD). Ann Intensive Care déc. 2015;5(1):58.

7. Parienti J-J, Mongardon N, Mégarbane B, Mira J-P, Kalfon P, Gros A, et al. Intravascular complications of central venous catheterization by insertion site. N Engl J Med. 2015;373(13):1220-9.

8. Wyatt CM, Vassalotti JA. We still go for the jugular: implications of the 3SITES central venous catheter study for nephrology. Kidney Int. 2016;89(18):522-4.

9. Parienti J-J, Thirion M, Mégarbane B, Souweine B, Ouchikhe A, Polito A, et al. Femoral vs jugular venous catheterization and risk of nosocomial events in adults requiring acute renal replacement therapy: a randomized controlled trial. JAMA. 2008;299(8):2413-22.

10. Parienti J-J, Mégarbane B, Fischer M-O, Lautrette A, Gazui N, Marin N, et al. Catheter dysfunction and dialysis performance according to vascular access among 736 critically ill adults requiring renal replacement therapy: a randomized controlled study. Crit Care Med avr. 2010;38(7):1118-25.

11. Oliver MJ, Callery SM, Thorpe KE, Schwab SJ, Churchill DN. Risk of bacteremia from temporary hemodialysis catheters by site of insertion and duration of use: a prospective study. Kidney Int déc. 2000;58(6):2543-5.

12. Hryszko T, Brzosko S, Mazerska M, Malyszko J, Mysliwiec M. Risk factors of nontunneled noncuffed hemodialysis catheter malfunction. A prospective study. Nephron Clin Pract. 2004;96(2):c43-47.

13. O'Grady NP, Alexander M, Burns LA, Dellinger EP, Garland J, Heard SO, et al. Guidelines for the prevention of intravascular catheter-related infections. Am J Infect Control mai. 2011:39(15):S1-34.

14. Pronovost P. Interventions to decrease catheter-related bloodstream infections in the ICU: the Keystone Intensive Care Unit Project. Am J Infect Control. 2008;36(16):S171.

15. Bellomo R, Ronco C, Mehta RL, Asfar P, Boisramé-Helms J, Darmon M, et al. Acute kidney injury in the ICU: from injury to recovery: reports from the 5th Paris International Conference. Ann Intensive Care. 2017

16. ACUTE KIDNEY INJURY | KDIGO. at http://kdigo.org/home/guidelines/ acute-kidney-injury/.

17. Huriaux L, Costille P, Quintard H, Journois D, Kellum JA, Rimmelé T. Haemodialysis catheters in the intensive care unit. Anaesth Crit Care Pain Med. 2017;36(5):313-9.

18. Gaudry S, Hajage D, Schortgen F, Martin-Lefevre L, Pons B, Boulet E, et al. Initiation strategies for renal-replacement therapy in the intensive care unit. N Engl J Med. 2016;375(19):122-33.

19. Mermel LA, Allon M, Bouza E, Craven DE, Flynn P, O'Grady NP, et al. Clinical practice guidelines for the diagnosis and management of intravascular catheter-related infection: 2009 update by the Infectious Diseases Society of America. Clin Infect Dis. 2009;49(1):1-45.

20. Fitzmaurice GM, Lipsitz SR, Ibrahim JG. A note on permutation tests for variance components in multilevel generalized linear mixed models. Biometrics. 2007;63(3):942-6.

21. Austin PC. An introduction to propensity score methods for reducing the effects of confounding in observational studies. Multivar Behav Res mai. 2011:46(3):399-424.

22. Fine JP, Gray RJ. A proportional hazards model for the subdistribution of a competing risk. J Am Stat Assoc. 1999;94(446):496-509.

23. Dugué AE, Levesque SP, Fischer M-O, Souweine B, Mira J-P, Megarbane B, et al. Vascular access sites for acute renal replacement in intensive care units. Clin J Am Soc Nephrol. 2012;7(1):70-7.

24. Bellomo R, Mårtensson J, Lo S, Kaukonen K-M, Cass A, Gallagher M, et al. Femoral access and delivery of continuous renal replacement therapy dose. Blood Purif. 2016;41(1-3):11-7.
25. Ng Y-H, Ganta K, Davis H, Pankratz VS, Unruh M. Vascular access site for renal replacement therapy in acute kidney injury: a post hoc analysis of the ATN study. Front Med. 2017:4:40.

26. Mrozek N, Lautrette A, Aumeran C, Laurichesse H, Forestier C, Traoré O, et al. Bloodstream infection after positive catheter cultures: what are the risks in the intensive care unit when catheters are routinely cultured on removal? Crit Care Med. 2011;39(6):1301-5.

27. Timsit J-F, Lugosi M, Minet C, Schwebel C. Should we still need to systematically perform catheter culture in the intensive care unit? Crit Care Med. 2011;39(6):1556-8.

28. Maki DG, Kluger DM, Crnich CJ. The risk of bloodstream infection in adults with different intravascular devices: a systematic review of 200 published prospective studies. Mayo Clin Proc. 2006;81(20):1159-71.

29. Cheng S, Xu S, Guo J, He Q, Li A, Huang L, et al. Risk factors of central venous catheter-related bloodstream infection for continuous renal replacement therapy in kidney intensive care unit patients. Blood Purif. 2018;28:1-8.

30. Souweine B, Lautrette A, Gruson D, Canet E, Klouche K, Argaud L, et al. Ethanol lock and risk of hemodialysis catheter infection in critically ill patients. A randomized controlled trial. Am J Respir Crit Care Med. 2015;191(9):1024-32.

31. Eggimann P, Pagani J-L, Dupuis-Lozeron E, Ms BE, Thévenin M-J, Joseph C, et al. Sustained reduction of catheter-associated bloodstream infections with enhancement of catheter bundle by chlorhexidine dressings over 11 years. Intensive Care Med. 2019:45(6):823-33.

32. Blot S, Poulakou G, Timsit J-F. Catheter-associated bloodstream infection rates: how low can you go? Intensive Care Med. 2019;45(6):896-7.

33. Timsit J-F, Dubois Y, Minet C, Bonadona A, Lugosi M, Ara-Somohano C, et al. New materials and devices for preventing catheter-related infections. Ann Intensive Care. 2011;1:34.

34. Timsit J-F, Rupp M, Bouza E, Chopra V, Kärpänen T, Laupland K, et al. A state of the art review on optimal practices to prevent, recognize, and manage complications associated with intravascular devices in the critically ill. Intensive Care Med. 2018;44(6):742-59.

35. Nuckols TK, Keeler E, Morton SC, Anderson L, Doyle B, Booth M, et al. Economic evaluation of quality improvement interventions for bloodstream infections related to central catheters: a systematic review. JAMA Intern Med. 2016;176(12):1843-54.

36. Mrozek N, Lautrette A, Timsit J-F, Souweine B. How to deal with dialysis catheters in the ICU setting. Ann Intensive Care. 2012;2(1):48.

37. Maizel J, Bastide M-A, Richecoeur J, Frenoy E, Lemaire C, Sauneuf B, et al. Practice of ultrasound-guided central venous catheter technique by the French intensivists: a survey from the BoReal study group. Ann Intensive Care. 2016;6(1):76

38. Rabindranath KS, Kumar E, Shail R, Vaux E. Use of real-time ultrasound guidance for the placement of hemodialysis catheters: a systematic review and meta-analysis of randomized controlled trials. Am J Kidney Dis. 2011;58(21):964-70.

39. Brass P, Hellmich M, Kolodziej L, Schick G, Smith AF. Ultrasound guidance versus anatomical landmarks for internal jugular vein catheterization. Cochrane Database Syst Rev. 2015;1:CD006962.

40. Saugel B, Scheeren TWL, Teboul J-L. Ultrasound-guided central venous catheter placement: a structured review and recommendations for clinical practice. Crit Care Lond Engl. 2017;21(1):225.

\section{Publisher's Note}

Springer Nature remains neutral with regard to jurisdictional claims in published maps and institutional affiliations. 